\title{
Effect of Ganoderma lucidum on MPTP Induced Behavioral Alterations in Swiss Albino Mice
}

\author{
Palanimuthu Vasanth Raj', Subramani Parasuraman² ${ }^{*}$, Sokkalingam Arumugam Dhanaraj', Lee Xin Ying ${ }^{3}$, Tan Hung Luc ${ }^{3}$, Stephanie A/P \\ Sandanasamy ${ }^{3}$, Koay Chin Yin ${ }^{3}$ \\ 'Pharmaceutical Technology Unit, Faculty of Pharmacy, AIMST University, Jalan Bedong Semeling, 08100, Bedong, Kedah Darul Aman, MALAYSIA. \\ 2Pharmacology Unit, AIMST University, Jalan Bedong Semeling, 08100, Bedong, Kedah Darul Aman, MALAYSIA. \\ UUndergraduate Pharmacy Student, AIMST University, Jalan Bedong Semeling, 08100, Bedong, Kedah Darul Aman, MALAYSIA.
}

\begin{abstract}
Objectives: To investigate the behavioral effect of Ganoderma lucidum polysaccharides (GLPS) and triterpenoid fractions (GLTT) in Parkinson's induced mice. Methods: Parkinson's disease (PD) was induced by intraperitoneal administration of of $3 \mathrm{mg} / \mathrm{kg}$ 1-methyl-4-phenyl-1,2,3,6-tetrahydropyridine (MPTP). G. lucidum was collected, authenticated and extracted with water and methanol to obtain GLPS and GLTT extracts respectively. A dose of $50 \mathrm{mg} / \mathrm{kg}$ of GLPS and GLTT were administered orally to PD mice for five consecutive days. Behavioral studies such as forced swim test, rota-rod test, grip strength test and locomotor activity were conducted in all the animals before and after toxicant, GLPS and GLTT treatment. Results: The mice injected with MPTP induced reasonable extent of Parkinsonism. In forced swim test, after treatment with GLPS and GLTT, the time for the mice to stop swimming decreased. In grip strength test, the motor coordination of PD induced mice seemed to be higher when compared to the normal mice, GLPS and GLTT treated groups. In rota-rod test, treatment with GLPS and GLTT showed mild decrease in the time for the mice to fall from a rotating rod. The results of the locomotor test
\end{abstract}

showed that the mice had an increase in its locomotor activity after being induced with MPTP and decreased after being treated with GLPS and GLTT. Conclusion: This preliminary study results indicate that both GLPS and GLTT extract has therapeutic benefit on the PD mice.

Key words: 1-methyl-4-phenyl-1,2,3,6-tetrahydropyridine (MPTP), Antiparkinsonism activity, Ganoderma lucidum, Polysaccharide, Triterpenoid.

Correspondence :

S. Parasuraman, M. Pharm,. Ph.D.,

Faculty of Pharmacy, AIMST University, Jalan Bedong Semeling, 08100, Bedong Kedah Darul Aman, MALAYSIA.

Office: +6044298000; Fax: +6044298009

E-mail: parasuphd@gmail.com

DOI: 10.5530/jyp.2016.3.5

\section{INTRODUCTION}

Parkinson's Disease (PD) is the most common progressive neurodegenerative movement disorder which affects around 6 million people globally and the prevalence will rise with an aging population. ${ }^{1,2}$ Majority of the cases of PD are still considered idiopathic. There are some hypotheses of neuronal loss which involves genetically linked factors and environmental interaction factors. Ingestion or inhalation of neurotoxins (1-methyl-4-phenyl-1,2,3,6-tetrahydropyridine [MPTP]), endogenous toxin releasing radical oxygen species (ROS) in brain and isoquinoline derivatives substances may cause $\mathrm{PD} .^{3}$

Although various therapeutic agents are available to cure PD symptomatically, there are many adverse effects associated with those medications. Taken levodopa as an example, even though it remains the pharmacologic mainstay for the treatment of $\mathrm{PD},{ }^{4}$ after several years of favorable responses, disabling motor complications frequently developed. ${ }^{5}$ Other than that, numerous side effects and complications of other dopaminergic medications and surgery had been testified too. ${ }^{6,7}$ So, these have reached our consensus that, till date, none of the drug or therapies has been convincingly shown to halt or cure the degenerative process in PD. In light of these challenges, researches into alternative neuro-protective therapies area are rising at a feverish pace. Increasing number of patients are seeking for alternative treatments such as herbal therapies. ${ }^{8}$ Many researchers had discovered the broad therapeutic potential of a mushroom species Ganoderma lucidum (Family: Ganodermataceae). Among the bioactive elements in G. lucidum, polysaccharides, triterpenes, and peptidoglycans are three major physiologically active constituents in G. lucidum. ${ }^{9,10}$ G. lucidum polysaccharides (GLPS) obtained by aqueous extraction are reported to exhibit a wide-ranging bioactivities, including anti-inflammatory, hypoglycemic, anti-ulcer, anti-tumorigenic, and immunostimulating effects. ${ }^{11,12}$ On the other hand, the triterpenoids extract of G. lucidum (GLTT) by methanolic extraction demonstrated antipruritic effects ${ }^{13}$ and anti-cancer properties ${ }^{14,15}$ Previous studies which stated that the extract of G. lucidum is effective to improve the function of mitochondria in aged rat brain, suggested it as a possible therapeutic application against ageing-associated neurodegenerative diseases. ${ }^{16}$ Of this interest, we hypothesize that G. lucidum extract has its therapeutic efficacy on PD. By using MPTP, PD was induced to Swiss albino mice, after which the effects of G. lucidum polysaccharide (GLPS) and triterpenoid (GLTT) fractions were investigated.

\section{MATERIALS AND METHODS}

G. lucidum was gifted by DXN Pharma Sdn Bhd., Malaysia. 1-methyl4-phenyl-1,2,3,6-tetrahydropyridine (MPTP) was obtained from Sigma Aldrich Co, St. Louis, USA. All chemicals, consumables and solvents used in this project were of analytical grade and purchased from Bendosen laboratory chemicals, Malaysia. EDTA, diethyl ether and formaldehyde were procured from RM chemicals/ HmbG chemicals, Malaysia.

\section{Animals}

Healthy, adult, both the gender of Swiss albino mice weighing 20-30 g were obtained from Central Animal house, AIMST University, Malaysia. The animals were housed in large, spacious poly acrylic cages at an ambient room temperature with $12 \mathrm{~h}$ light and dark cycle. The animals were fed with water and food ad libitum. The PD induced animal model study was approved by AIMST University Human and Animal Ethics Committee and the study was conducted according to Animal Research Review Panel guidelines. 


\section{Extraction of GLPS and GLTT}

G. lucidum was extracted with distilled water by maceration in a dark place for 6 days. Few drops of 1\% chloroform were used as preservative to prevent fungal growth. Later, the extract was filtered and dried at $60^{\circ} \mathrm{C}$. The semisolid form of G. lucidum polysaccharide (GLPS) extract was preserved in cold conditions until further studies.

For the triterpenoid extraction, G. lucidum powder extracted with methanol by Soxhlet extraction for $24 \mathrm{~h}$. The extract was evaporated to dryness on a rotary evaporator to obtain G. lucidum triterpenoid (GLTT). The extract was stored in cold conditions until further studies.

\section{Antiparkinson activity of GLPS and GLTT}

Healthy Swiss albino mice were divided into five groups each of five animals as follows.

Group I : Control

Group II : MPTP control

Group III : MPTP+GLPS $50 \mathrm{mg} / \mathrm{kg}$

Group IV : MPTP+GLTT 50 mg/kg

A control group animal were administered with normal saline $(1 \mathrm{ml} /$ $\mathrm{kg}$ ) and the animals in group II to IV were administered with $3 \mathrm{mg} /$ $\mathrm{kg}$ MPTP (dissolved in saline), intraperitoneally for 5 consecutive days. After induction of PD, the animals in group III and IV were treated with GLPS (50 mg/kg) and GLTT (50 mg/kg). The drugs were suspended with $0.5 \%$ carboxymethyl cellulose and administered orally for 5 consecutive days. During the experiment, body weight variations, regular food and water intake was measured. Prior to the experiment and at the end of the experiment, the rats' behavior was studied using forced swim test, grip strength test, rota-rod test and locomotor activity. ${ }^{17,18}$ At the end of the study the rats were sacrificed and brain samples were collected and preserved in $10 \%$ neutral formalin for histopathological studies.

Forced swim test: In this test, the tank with water of temperature $24^{\circ} \mathrm{C}-30^{\circ} \mathrm{C}$ with depth that the rodent's tails and feet not touching the bottom of the tank was prepared. The rodent was then put into the tank upon which the time between the rodent to stop swimming and start sinking was recorded. The rodent was removed immediately as soon as it started to sink. The procedure was repeated for all the rodents. The water tank was then cleaned as the accumulation of faeces and urine could cause bacterial contamination.

Grip strength test: The forelimb of the rodent was left to cling on a rope while its body and tail was suspended in the air above $50 \mathrm{~cm}$ from the ground. The time of the rodent clinging on the rope was recorded. The procedure was repeated for all 5 rodents of both control and treated group. The height between the rope and ground was ensured not be too high, to prevent the rodent hurt when falling.

Rota-rod test: The rodent was placed on a rota-rod with speed of 20 RPM. The time between rodent maintain on the rota-rod until it fall was recorded. The procedure was repeated for each rodent.
Locomotor activity: The locomotor activity of the rats was recorded in actophotometer provided with an acrylic cage and 8 beams of infrared light along both the $\mathrm{x}$ - and $\mathrm{y}$-axis. The activity of each rat was monitored at room temperature over $10 \mathrm{~min}$.

\section{Histopathological analysis}

The brain samples were preserved in $10 \%$ neutral formalin for histopathological analysis. The brain sample were embedded in paraffin after being dehydrated in alcohol and subsequently cleared with xylene. A 5-6 micrometer thickness of brain section were prepared from paraffin blocks and stained with hematoxylin and eosin and mounted in neutral DPX medium, and the sections were examined under light microscope.

\section{Statistical analysis}

All statistical data are represented as mean \pm SEM of the mentioned number of testing in five animals. One way ANOVA (Graph Pad Prism 5.00, Instat software, San Diego, CA< USA) followed by Tukey's post hoc test were used for the statistical analysis.

\section{RESULT}

The semisolid form of G. lucidum polysaccharide (GLPS) and triterpenoid (GLTT) extracts were used in this study. The percentage yield of GLPS and GLTT were found to be $8.88 \%$ and $6.67 \%$ respectively.

The effect of GLPS and GLTT extract on MPTP induced behavioral alterations were summarized in Table 1. On day of termination MPTP treated animals showed increased swimming ability, muscular strength, and increased locomotor activity. Whereas GLPS and GLTT significantly inhibited the MPTP induced behavioral alterations. No significant alteration was observed in rota-rod test. Histopathological analysis did not indicate any abnormality in the brain tissue of GLPS and GLTT treated animals (Figure 1).

\section{DISCUSSION}

Parkinson's disease is characterized by the tetrad of tremor at rest, rigidity, decreased voluntary movements, and postural instability due to depletion of dopamine levels in nigrostriatal system and loss of neurons of nigrostriatal dopaminergic pathway. loss of dopamine producing neurons in the nigrostriatal system. The mice were injected with MPTP to induce PD, because the pathogenesis of MPTP induced PD in mice is clearly linked to a form of human parkinsonism. ${ }^{19,20}$ Since GLPS and GLTT, has combination benefit without toxicity which represents the desired end results in the development of effective therapeutics interventions, it has been used for hundreds of years as a health promotion and treatment strategy. ${ }^{21,22}$

In the present study, MPTP administered group showed an increased neuronal and muscular activities rather than a decrease. PD was char-

Table 1: Behavioral studies of PD induced mice models before and after GLPS and GLTT treatment

\begin{tabular}{cccccc}
\hline Behavioral Tests & $\begin{array}{c}\text { Baseline } \\
\text { (seconds) }\end{array}$ & $\begin{array}{c}\mathbf{1}^{\text {st }} \text { after } \\
\text { (seconds) }\end{array}$ & $\begin{array}{c}5^{\text {th }} \text { day } \\
\text { after MPTP } \\
\text { (seconds) }\end{array}$ & $\begin{array}{c}\text { GLPS 50 mg/kg } \\
\text { (seconds) }\end{array}$ & $\begin{array}{c}\text { GLTT 50 mg/kg } \\
\text { (seconds) }\end{array}$ \\
\hline Forced Swim Test & $84.00 \pm 1.44$ & $118.00 \pm 4.98$ & $158.20 \pm 5.51$ & $57.20 \pm 9.83$ & $60.50 \pm 6.10$ \\
Grip Strength Test & $89.20 \pm 1.48$ & $120.20 \pm 4.73$ & $160.80 \pm 5.20$ & $81.60 \pm 4.40$ & $90.10 \pm 5.40$ \\
Rota-rod Test & $25.60 \pm 3.65$ & $25.20 \pm 1.37$ & $29.10 \pm 2.57$ & $17.70 \pm 3.39$ & $19.50 \pm 2.84$ \\
Locomotor activity & $79.00 \pm 1.12$ & $118.00 \pm 2.00$ & $136.00 \pm 1.77$ & $108.00 \pm 4.12$ & $142.00 \pm 2.98$ \\
\hline
\end{tabular}

*The values are mean \pm SEM of five animals. 


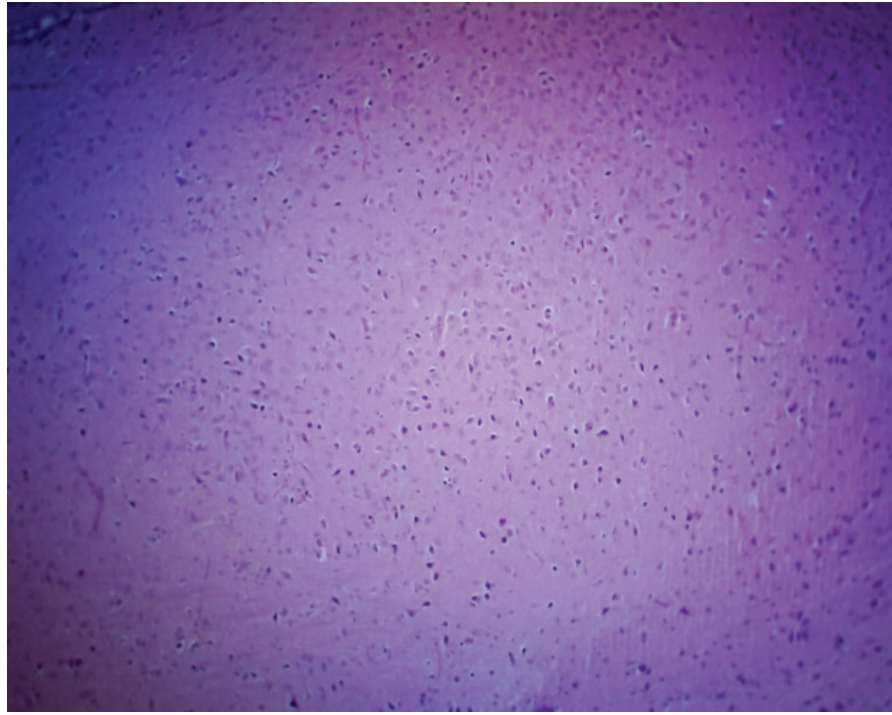

Figure 1(A): A Section from the brain of a normal animal which shows partial distruction of neuronal cells. $\mathrm{H}$ and $\mathrm{E}, \times 100$.

acterized by a decreased voluntary movement, but we observed an increased motor activity after MPTP administration and this may be due to its sympathomimetic over activation or frequency of MPTP which may not be sufficient to induce PD in experimental animals. Porras et al induced PD in monkey and mouse and they found that "MPTP mouse models for instance failed to replicate symptomatic manifestation of PD".22 GLPS and GLTT were found to alter the MTPT induced increase in behavioral function and this indicates that both GLPS and GLTT has some degree of neuroprotective effect. Zhang et al., and Zhou et al., studied the neuroprotective effect of G. lucidum and the effect is due to reduction in the percentage of apoptotic neurons, suppressed overexpression of active caspasses- $3,-8$ and -9 , myeloperoxidase activity, malondialdehyde levels, nitric oxide levels and inhibition of the reduction of Bcl-2 expression. . $^{23,24}$

\section{CONFLICT OF INTEREST}

All authors declare no conflict of interest.

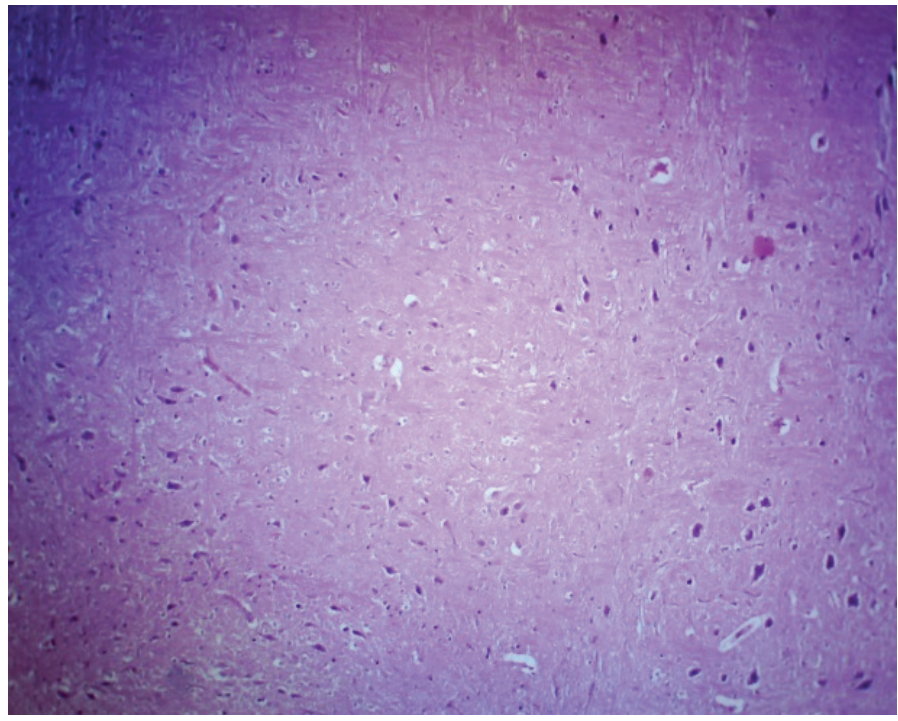

Figure 1(B): A Section from the brain of an MPTP adminsited animals shows partial distruction of neuronal cells. $\mathrm{H}$ and $\mathrm{E}, \times 100$.

\section{CONCLUSION}

The mice models injected with MPTP successfully induced reasonable extent of Parkinsonism. After employing GLPS and GLTT into the PD induced mice models, the behavioral studies of forced swim test and grip strength test reflected mean values which indicated that GPLS and GLTT held therapeutic benefit on the mice models, when compared with control.

\section{ACKNOWLEDGEMENTS}

The authors wish to thank AIMST University, Malaysia for providing research facilities and FRGS grant (FRGS/2/2013/SG05/AIMST/01/1) on 'Neural stem cell gene therapy for cell signaling in PD induced model in mice' as a support. Also sincerely thank DXN Pharma Sdn Bhd., Malaysia for providing us pure sample of G. Lucidum.

\section{ABBREVIATIONS USED}

PD: Parkinson's disease; MPTP: (1-methyl-4-phenyl-1,2,3,6-tetrahydropyridine ); GLPS: Ganoderma lucidum polysaccharides; GLTT: Ganoderma lucidum triterpenoid; ROS: Radical oxygen species.

\section{ABOUT AUTHORS}

Palanimuthu Vasanth Raj: Is an Associate professor in the unit of Pharmaceutical Technology, Faculty of Pharmacy, AIMST University, Malaysia. His research interest is in the areas of cancer biology, gene expression analysis and nano-formulations.

Subramani Parasuraman: Is a Senior Lecturer, Unit of Pharmacology, Faculty of Pharmacy, AIMST University, Malaysia. His research interest is in the areas of pre-clinical pharmacodynamics and toxicological studies. 


\section{REFERENCES}

1. Baker MG, Graham L. The Journey: Parkinson's disease. BMJ. 2004;329(7466):6114.

2. Reeve A, Simcox E, Turnbull D. Ageing and Parkinson's disease: why is advancing age the biggest risk factor?. Ageing Res Rev. 2014;14:19-30.

3. Dauer W, Przedborski S. Parkinson's disease: mechanisms and models. Neuron. 2003;39(6):889-909.

4. Silva MA, Mattern C, Häcker R, Tomaz C, Huston JP, Schwarting RK. Increased neostriatal dopamine activity after intraperitoneal or intranasal administration of L-DOPA: on the role of benserazide pretreatment. Synapse. 1997;27(4):294-302.

5. Lesser RP, Fahn S, Snider SR, Cote LJ, Isgreen WP, Barrett RE. Analysis of the clinical problems in parkinsonism and the complications of long-term levodopa therapy. Neurology. 1979;29(9 Pt 1):1253-60.

6. 2012 Consensus guidelines for the treatment of Parkinson's disease. Available in http://www.neuro.org.my/MSN GUIDELINE/MSN_GUIDELINE_PD-Guidelines 2012_revised08Jan2013.pdf [Last accessed on 31/03/2016]

7. Pepper J, Zrinzo L, Mirza B, Foltynie T, Limousin P, Hariz M. The risk of hardware infection in deep brain stimulation surgery is greater at impulse generator replacement than at the primary procedure. Stereotact Funct Neurosurg. 2013;91(1):56-65.

8. Suchowersky O, Gronseth G, Perlmutter J, Reich S, Zesiewicz T, Weiner WJ. Quality Standards Subcommittee of the American Academy of Neurology. Practice Parameter: neuroprotective strategies and alternative therapies for Parkinson disease (an evidence-based review): report of the Quality Standards Subcommittee of the American Academy of Neurology. Neurology. 2006;66(7):976-82.

9. Boh B, Berovic M, Zhang J, Zhi-Bin L. Ganoderma lucidum and its pharmaceutically active compounds. Biotechnol Annu Rev. 2007;13:265-301.

10. Zhou X, Lin J, Yin Y, Zhao J, Sun X, Tang K. Ganodermataceae: Natural products and their related pharmacological functions. Am J Chin Med. 2007;35(4):55974.

11. Bao X, Liu C, Fang J, Li X. Structural and immunological studies of a major polysaccharide from spores of Ganoderma lucidum (Fr.) Karst. Carbohydr Res. 2001;332(1):67-74.

12. Wachtel-Galor S, Yuen J, Buswell JA, Benzie IFF. (2011) Ganoderma lucidum (Lingzhi or Reishi): A Medicinal Mushroom. Herbal Medicine: Biomolecular and Clinical Aspects. $2^{\text {nd }}$ edition. Boca Raton (FL): CRC Press: Chapter 9.
13. Andoh T, Zhang Q, Yamamoto T, Tayama M, Hattori M, Tanaka K, et al. Inhibitory effects of the methanol extract of Ganoderma lucidum on mosquito allergyinduced itch-associated responses in mice. J Pharmacol Sci. 2010;114(3):292-7.

14. Thyagarajan A, Jedinak A, Nguyen $H$, Terry $C$, Baldridge LA, Jiang J, et al. Triterpenes from Ganoderma lucidum induce autophagy in colon cancer through the inhibition of p38 mitogen-activated kinase (p38 MAPK). Nutr Cancer. 2010;62(5):630-40.

15. Vasanth RP, Dhanaraj SA, Ali SA, Kuan TH, Mathiyalagan S, Raman S. Anti-proliferative effect of Ganoderma lucidum polysaccharide and triterpenoid fractions against cancer cells. Manipal J Pharm Sci. 2015 |;1(1):32-7.

16. Ajith TA, Sudheesh NP, Roshny D, Abishek G, Janardhanan KK. Effect of Ganoderma lucidum on the activities of mitochondrial dehydrogenases and complex I and II of electron transport chain in the brain of aged rats. Exp Gerontol. 2009;44(3):219-23.

17. Chen PC, Vargas MR, Pani AK, Smeyne RJ, Johnson DA, Kan YW. Nrf2-mediated neuroprotection in the MPTP mouse model of Parkinson's disease: Critical role for the astrocyte. Proc Natl Acad Sci U S A. 2009;106(8):2933-8.

18. Parasuraman S, Sujithra J, Syamittra B, Yeng WY, Ping WY, Muralidharan S, et al. Evaluation of sub-chronic toxic effects of petroleum ether, a laboratory solvent in Sprague-Dawley rats. J Basic Clin Pharm. 2014;5(4):89-97.

19. Jackson-Lewis V, Przedborski S. Protocol for the MPTP mouse model of Parkinson's disease. Nat Protoc. 2007;2(1):141-51.

20. Dauer W, Przedborski S. Parkinson's disease: mechanisms and models. Neuron. 2003;39(6):889-909

21. Wachtel-Galor S, Buswell J. A, Tomlinson B, Benzie IFF. Lingzhi polyphorous fungus. In: Herbal and Traditional Medicine: Molecular Aspects of Health. New York: Marcel Dekker Inc. 2004. pp. 179-228.

22. Porras G, Li Q, Bezard E. Modeling Parkinson's disease in primates: The MPTP model. Cold Spring Harb Perspect Med. 2012;2(3):a009308.

23. Zhang W, Zhang Q, Deng W, Li Y, Xing G, Shi X, Du Y. Neuroprotective effect of pretreatment with Ganoderma lucidum in cerebral ischemia/reperfusion injury in rat hippocampus. Neural Regen Res. 2014;9(15):1446-52.

24. Zhou ZY, Tang YP, Xiang J, Wua P, Jin HM, Wang Z, et al. Neuroprotective effects of water-soluble Ganoderma lucidum polysaccharides on cerebral ischemic injury in rats. J Ethnopharmacol. 2010;131(1):154-64. 